\title{
How does the brain process music?
}

\author{
Jason Warren
}

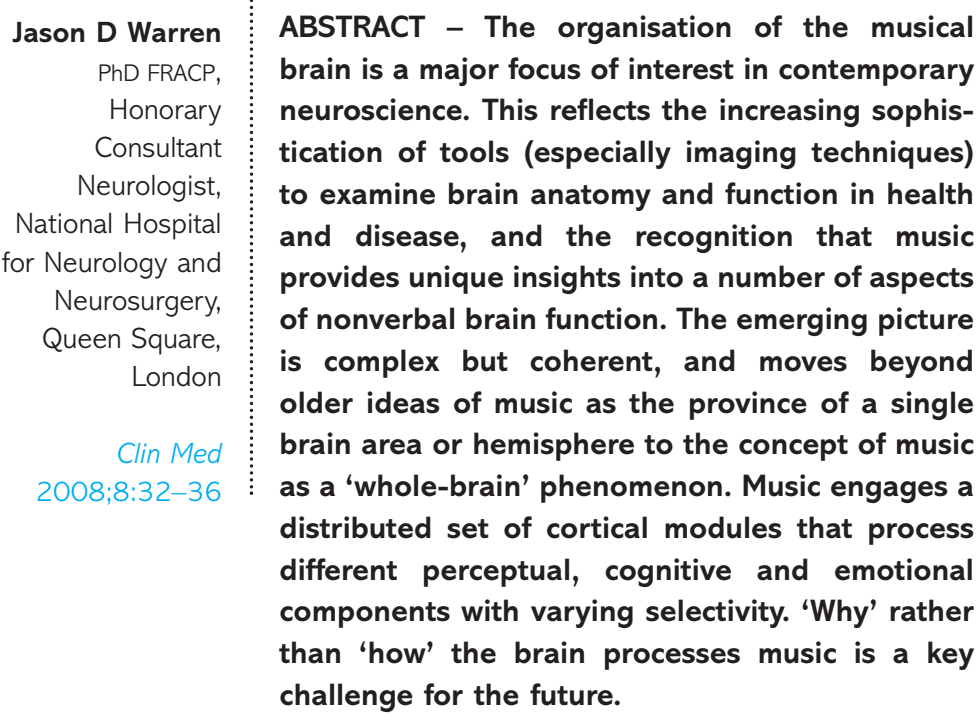

All normal human brains can recognise music. How does the brain do it? The apparent universality of music implies that our musical experience, in all its exuberant variety, is underpinned by certain basic processes that reflect the anatomical and physiological organisation of our brains. It follows that the perception of music (like other higher brain functions) may be susceptible to specific disease states and brain lesions. Recent progress in the basic and clinical neurosciences, notably structural and functional brain imaging, have confirmed these predictions. ${ }^{1,2}$ In this piece I draw on some of these approaches to outline a framework for the organisation of the musical brain. I use 'musical brain' here to refer to the brain areas and mechanisms that enable any normal listener to perceive and understand music, rather than the brain specialisations that may support music perception and production in individuals with musical training.

\section{Problems in studying the musical brain}

The neuroscientific study of music poses a number of problems. These reflect the nature of music (and musicality) itself, prevailing concepts about it, and the tools available to study it.

It is stating the obvious to say that music is not simple: even a single voice melody has multiple dimensions of pitch (the individual intervals, and the overall pattern of 'ups and downs'), time (tempo, rhythm, metre) and the distinctive instrumental or human voices ('tone quality' or timbre) that carries the tune. It is not obvious a priori how these dimensions might translate to brain organisation, though it would seem unlikely that a single 'music centre' processes them all.

The problems this entails are not unlike those confronting the study of that other uniquely human, multidimensional capacity, language. Like language, music is an abstract, rule-based system (arguably the only one of comparable complexity), and the parallels between music and language are seductive. It is possible to devise musical analogies for linguistic elements such as 'vocabulary' and 'grammar', and at a clinical level, to find musical equivalents for the aphasias. The analogies between music and language are, however, superficial and quickly raise thorny issues. It is no evolutionary accident that we do not use music to prepare a shopping list, while the kind of information that music can uniquely convey remains a rich source of philosophical controversy. That musical and verbal capacities sometimes dissociate (exemplified by the patient with expressive aphasia who remains able to sing) is a timehonoured observation of clinical neurology. However, the brain imaging sciences have dispelled the traditional notion of music and language as 'mirror' phenomena represented in opposite cerebral hemispheres.

Finally (and in pointed contrast to language), musical attainment varies widely in the population at large and is difficult to measure. Disorders of music processing rarely occur in isolation, and it may not be possible to evaluate musical functions reliably if there is a significant associated aphasia or other cognitive deficits. Such difficulties have led to a considerable bias in the clinical literature, since musical disorders are generally characterised only when they occur as relatively circumscribed deficits in musically competent individuals. This factor may partly account for the preponderance of right hemispheric lesions reported to produce musical deficits, since individuals with left hemisphere damage are often aphasic. The extent to which the brain organisation for music in trained musicians is representative of the wider population is, of course, moot. Coupled with this, the anatomy of music perception (and indeed, cognitive processes in general) was, until comparatively recently, something of a black box, 
deduced largely from clinical observations of the effects of strategic brain lesions.

\section{Scientific approaches to the musical brain}

A number of recent developments have led to a re-evaluation of the musical brain. ${ }^{1,2}$ Firstly, and arguably most importantly, models of music processing have been developed that do justice to its complex multidimensionality. ${ }^{1-7}$ These models were based initially on accumulated neurological case studies documenting the musical deficits exhibited by patients with strokes and other forms of focal brain damage. Impairments of particular musical functions that leave other functions intact are especially telling: such deficits illustrate the more general principle of dissociation, implying functional and anatomical specificity.

Acquired brain defects of music processing or 'amusias' are often associated with other central auditory processing deficits (for example, agnosia for environmental sounds), suggesting that the processing resources for different types of complex sounds are at least partly in common., ${ }^{2,8}$ Moreover, the amusias share certain neuropsychological characteristics with complex perceptual disorders in other sensory domains (such as vision), suggesting that the principles of brain organisation are broadly similar. For example, the ability to discriminate melodies may be affected despite retained recognition of familiar melodies: an 'apperceptive agnosia' for music. ${ }^{6,2}$ The converse pattern corresponds to an 'associative agnosia' for music. More fine-grained deficits correspond to relatively selective impairments of cortical modules that process particular perceptual components of music, such as pitch or melody ('dysmelodia'), rhythm ('dysrhythmia') or timbre ('dystimbria'). ${ }^{2}$ Besides identifying brain processing modules that support different musical functions, the pattern of musical deficits observed between patients and in a single patient over time (for example, in the recovery phase of stroke) can suggest how these modules are related to one another functionally and anatomically (Fig 1). Reliance on identification of rare patients presenting with symptomatic musical deficits (the 'symptom-led' approach) has been amplified by the systematic study of series of patients with brain lesions of a particular kind (the 'lesion-led' approach), most often stroke or temporal lobectomy (for refractory epilepsy). Neither approach is entirely satisfactory. The symptom-led approach is vulnerable to ascertainment bias and lesion heterogeneity, while the lesion-led approach does not resolve the clinical significance of culprit lesions.

Neuropsychological instruments to assess musical functions, such as the Montreal Battery for the Evaluation of Amusia, ${ }^{9}$ have provided normative data about music perception in the general population and assist in quantifying and comparing the effects of brain damage. Brain lesions can now be defined precisely using structural magnetic resonance imaging (MRI). More subtle changes in brain structure can be measured at a group level using statistically motivated techniques such as voxel-based morphometry, in order to assess structural changes that are common to different brains.

During the past two decades, the modular organisational scheme of music processing, suggested by the neuropsychological study of patients with brain damage, has been refined substantially by the application of brain imaging techniques (positron emission tomography and functional MRI) that can capture the function (as well as the anatomy) of the normal brain. This approach has helped define the brain basis for many of the processing modules predicted from neuropsychological work. ${ }^{1,7}$ The insights provided by anatomical lesion correlation and functional brain imaging are complementary: the former can identify critical sites for particular functions, while the latter can delineate the complete network that supports the function. The picture emerging from these different approaches is generally convergent, and they are accordingly considered together in the following sections. Rather than 'brain mapping' par excellence, contemporary imaging neuroscience emphasises the testing of specific hypotheses about brain organisation, an enterprise that will be guided by neuropsychologically informed models of music processing and by the increasing application of techniques to assess anatomical and functional connections between brain areas.

\section{A scheme for the organisation of the musical brain}

\section{Principles of central auditory processing}

The human auditory brain has a hierarchical organisation, anatomically and functionally: areas that are lower in the hierarchy pass information to higher stages (this traffic is rarely, if ever, exclusively one way), and increasingly complex and/or abstract features are represented at each stage. Music, as a species of complex sound, engages this hierarchy. Basic acoustic building blocks such as fundamental frequency, harmonics, and the duration and loudness of the individual notes in a melody are first encoded. Multiple successive processing stages represent the collections of perceptual features corresponding to a particular instrument or melody; disambiguate simultaneous instruments and melodies; link these representations with stored musical memories and knowledge; import information from other cognitive domains; and ultimately, programme an appropriate behavioural response. The emotional content of the music is analysed in a partly independent brain hierarchy that helps to direct the behavioural response and to determine future behaviour (such as music seeking or avoidance).

Like any sound, music is first processed in the ascending auditory pathway from the cochlea to the primary auditory cortex. This processing is essentially concerned with the coding of elementary attributes of individual sounds and sound components. The subsequent stages in the processing hierarchy require the cerebral cortex. The distinction between the subcortical and cortical stages of processing is illustrated by the example of pitch. Pitch can be represented as a physical frequency or repetition rate of an acoustic signal, however the perception of pitch does not depend on a single physical attribute (for example, the pitch of a harmonic series can be perceived even if the fundamental frequency corresponding to the pitch is missing). Coding of the acoustic correlates of pitch (such as the energy in 
a particular frequency band) occurs in the ascending auditory pathway, while the perception of pitch arises at the level of the cortex. ${ }^{4}$ The percept here 'emerges' as a result of cortical processing of the overall pattern of the acoustic signal, which could itself be created by various physical means. This type of processing is particularly relevant to music, where perceptual effects may rely on the emergence of patterns (eg voices in a fugue) that would be difficult to predict from a strictly acoustic analysis.
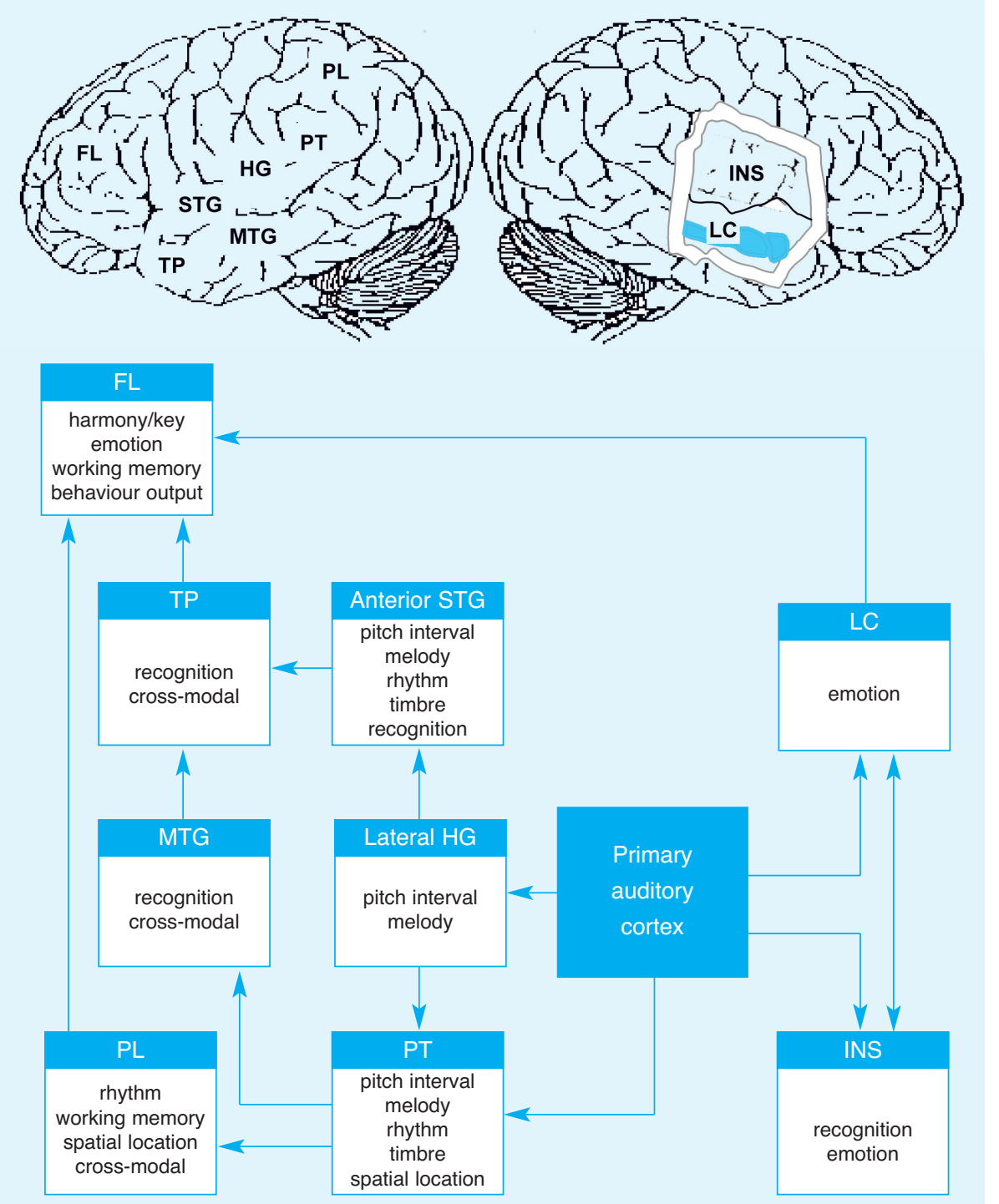

Fig 1. A scheme for the organisation of the musical brain based on evidence from the study of normal and damaged brains. Key brain areas are shown above and major functional associations of these areas are represented below. Arrows indicate the predominant flow of information between cortical areas (most of these connections are bidirectional). Overall there is a right hemisphere functional preponderance for processing a number of components of music, however this selectivity is relative rather than absolute, and a similar qualification applies to the processing of particular musical components by different brain areas within a hemisphere. Partly independent brain networks govern perceptual analysis and emotional response. The scheme indicates the broadly hierarchical nature of music processing, with more complex and abstract properties represented by areas further beyond primary auditory cortex. $\mathrm{FL}=$ frontal lobe; $\mathrm{HG}=$ Heschl's gyrus (site of primary auditory cortex); INS = insula (shown with overlying cortex removed); LC = limbic circuit (shown with overlying cortex removed); MTG = middle temporal gyrus; PL = parietal lobe; $\mathrm{PT}=$ planum temporale; $\mathrm{STG}=$ superior temporal gyrus; $\mathrm{TP}=$ temporal pole.

\section{Overview of cortical circuitry}

The human primary auditory cortex is situated in the medial tains a centre for the persure. The lateral portion of $\mathrm{HG}$ connetwork of higher cortical areas in the temporal, parietal and frontal lobes. This network includes auditory 'association areas' that process certain properties of complex sounds. Posterior to HG lies the planum temporale (corresponding in the left hemisphere to Wernicke's area). This is an auditory association area that has been implicated in the analysis of different attributes of complex sound sources, including their spatial location, identifying features (such as the acoustic pattern corresponding to a spoken syllable, or the timbre of a voice or musical instrument), and information about pitch patterns they carry. ${ }^{5,10}$ Anterior to HG is the superior temporal gyrus (STG) which is engaged in the analysis of streams of auditory information such as a spoken sentence or musical melody. ${ }^{4,5}$ Adjacent and surrounding areas in the anterior temporal lobe and insula are involved in identification of nonverbal sounds including familiar music. ${ }^{11,12}$ The lateral temporal and parietal lobes link auditory information with information derived from other sensory modalities (especially vision). Circuits in the parietal and frontal lobes mediate working memory for music and other sounds and behavioural responses to sound.

The processing of music and other complex nonverbal sounds thus engages a substantial proportion of the cortical mantle, rivalling the language system in breadth and complexity. ${ }^{13}$ The organisation of the musical brain is schematised in Fig 1. The evidence of brain imaging studies has demonstrated that music shares basic brain circuitry with other types of complex sound, and no single brain area can be regarded as exclusively dedicated to music. ${ }^{1,2,7}$ Conversely, the various perceptual and cognitive components of music are each processed by several brain areas, linked together in a functional hierarchy. Selectivity for music and for particular components of music is therefore relative rather than absolute, and it emerges in the pattern 
of activation in a network of brain areas. The musical brain is a super-structure comprising multiple overlapping processing modules, each formed by a network of anatomical and functional links between cortical areas. This overarching organisation is examined in more detail in the following section.

\section{Components of music}

Pitch. In music, pitch is used to construct melodies (patterns of pitch over time), chords (the simultaneous presentation of more than one pitch) and harmonies (the simultaneous presentation of more than one melody). Brain activity during the analysis of melodies occurs in the anterior and posterior superior temporal lobes bilaterally, typically with greater activation of the right hemisphere. ${ }^{14}$ Brain lesions that involve the superior temporal lobe in the right hemisphere tend to disrupt the perception of melodies more than comparable lesions of the left hemisphere. Though by no means absolute, this contrasts with the left hemisphere emphasis for the processing of verbal information. In Western tonal music, melodies are constructed using keys where only certain notes are allowed within every octave, and the analysis of key information involves additional areas in the medial frontal cortex. ${ }^{14}$ This dimension has no precise analogue in speech or other types of complex sound. However, certain aspects of pitch sequence processing such as expectancy and the violation of harmony involve the right hemisphere analogue of Broca's area in the inferolateral frontal lobe. ${ }^{15}$

The processing of different kinds of pitch information illustrates a further key theme in the organisation of the musical brain: the distinction between 'local' and 'global' levels of analysis. The 'local' level corresponds to the absolute values of the intervals between pitches in a melody, whereas the 'global' level corresponds to the pitch contour (the pattern of 'ups' and 'downs') defining the melody as a whole. Peretz showed that different sites of brain damage can differentially affect the use of local and global information in melodies. ${ }^{3}$ Patients with right cerebral hemisphere strokes could assess neither global nor local information, while patients with strokes involving the left hemisphere could use global but not local information. Isolated impairments of local processing but no isolated impairments of global processing were observed. This suggests a model in which the right hemisphere derives pitch contour and the left hemisphere uses this contour to fill in the detailed pitch interval structure. The validity of this model continues to be examined. ${ }^{16,17}$ However, the model underlines the importance of cooperation between the cerebral hemispheres in music processing: music is not the province of either hemisphere exclusively, rather the two hemispheres are relatively more or less involved in particular aspects of music analysis.

Time information. The brain mechanisms that process temporal structure in music (tempo, rhythm and metre) have been less investigated than those that underlie pitch perception. ${ }^{2}$ These elements could be regarded as a temporal 'hierarchy' somewhat analogous to pitch interval, melody and harmony in the pitch domain. Impaired detection of rhythmic changes has been described in left temporoparietal stroke and left hippocampal sclerosis, ${ }^{18}$ while other studies have not demonstrated laterality differences. $^{3,17}$ However, functional imaging studies have demonstrated activity in the lateral cerebellum and basal ganglia during the reproduction of a rhythm, and there may be distinct representations for sequences with time intervals in integer ratios (more common in music) compared with non-integer ratios. ${ }^{19}$ The observed activation of motor structures suggests that the perception and production of rhythm may share brain circuitry, though this is likely to apply to rhythm in other auditory and visual domains as well as music. The brain basis for metrical processing remains poorly defined, and indeed, this is difficult to assess reliably in musically naive subjects. In a temporal lobectomy series, Liegeois-Chauvel et al found metrical impairments following left and right anterior temporal lobe resections. ${ }^{16}$ Neither Ayotte et al ${ }^{11}$ nor Peretz ${ }^{3}$ found stroke patients with heterogeneous left and right hemisphere strokes to be impaired relative to neurologically normal control subjects, while Schuppert et al ${ }^{17}$ found that both left and right hemispheric stroke patients were impaired relative to controls.

Timbre. The perception of timbre has not been extensively studied, however amusia is frequently accompanied by an alteration in perceived quality of music (often described as unpleasant, 'flat' or 'mechanical' in nature). There may also be inability to recognise musical instruments. Right superior temporal lobe areas that overlap those implicated in melody analysis are critical for normal timbre perception. Timbral deficits are generally associated with pitch perception deficits, however selective 'dystimbria' may arise in association with lesions involving right STG. ${ }^{20,21}$

Meaning. Beyond the perceptual components of music, the brain basis for attributing meaning at the level of familiarity and recognition of pieces is less well established. Deficits in the recognition of familiar tunes may occur with damage involving the anterior STG and insula in either cerebral hemisphere and similar areas are activated in healthy subjects. ${ }^{11,12}$

Emotion. Partly in parallel to the extensive cortical network for the perceptual and cognitive processing of sound lies the phylogenetically much older circuit that mediates emotional responses. This circuit includes the amygdala, hippocampus, and their subcortical and cortical connections, collectively comprising the 'limbic system'. While many natural sounds have some emotional quality, this dimension assumes disproportionate importance in the case of music. Functional imaging work in healthy subjects has demonstrated that strong emotional responses to music are associated, paradoxically, with limbic activity very similar to that elicited by basic biological drives. $^{22}$ The affective and perceptual dimensions of music are dissociable; loss of pleasure in music can occur despite normal perceptual analysis, and vice versa. Altered emotional responses to music occur with lesions involving the right posterior temporal lobe and insula. The insula is a multimodal area that has been implicated in many aspects of perceptual, cognitive and emotion processing; it is therefore a good candidate site 
for the integration of cognitive and affective dimensions of the response to music. Reduced intensity of musical emotion may be associated with damage to either mesial temporal lobe, ${ }^{23,24}$ implicating the limbic areas predicted by functional imaging evidence. One patient with infarction of the left amygdala and insula no longer experienced 'chills' in response to Rachmaninov preludes. ${ }^{23}$ There may be a hierarchy of emotional responses analogous to those identified for other kinds of musical information: dissonant sounds are perceived as unpleasant by virtually all Western listeners, whereas 'chills' are highly subjective and may depend on more complex structural features of the music as well as the individual's personal musical experience.

\section{Conclusions}

How does the brain process music? The outline of an answer is now possible. The multiple acoustic dimensions of music are first encoded in the ascending auditory pathways to primary auditory cortex. Perceptual patterns corresponding to these dimensions are extracted by primary and higher auditory cortices, using a modular neural machinery that shows relative selectivity for particular perceptual components. This machinery is shared between the cerebral hemispheres. Each processing module represents increasingly complex properties at successive stages in a hierarchical network of linked cortical areas. Widely distributed areas beyond auditory cortex link perception with meaning and memory and mediate behavioural responses to music. Partly in parallel to the perceptual and cognitive processing hierarchy is the limbic circuitry via which music engenders the powerful and neurally primitive emotional responses that are fundamental to the quality of musical experience. Imperfect as it is, our emerging picture of the musical brain is remarkably coherent considering the many difficulties that confront the attempt to deconstruct music as a neurobiological phenomenon. Perhaps the single greatest challenge for the future will be to move beyond 'how' to ask 'why' the brain processes (indeed, creates) music. Why devote such elaborate resources to an abstract stimulus with no obvious survival value? The answer to that question would surely illuminate music and brain alike.

\section{Acknowledgements}

The author is supported by a Wellcome Trust Intermediate Clinical Fellowship.

\section{References}

1 Peretz I, Zatorre RJ. Brain organization for music processing. Annu Rev Psychol 2005;56:89-114.

2 Stewart L, Von Kriegstein K, Warren JD, Griffiths TD. Disorders of musical listening. Brain 2006;129:2533-53.

3 Peretz I. Processing of local and global musical information by unilateral brain-damaged patients. Brain 1990;113:1185-205.

4 Patterson RD, Uppenkamp S, Johnsrude IS, Griffiths TD. The processing of temporal pitch and melody information in auditory cortex. Neuron 2002;36:767-6.
5 Warren JD, Uppenkamp S, Patterson RD, Griffiths TD. Separating pitch chroma and pitch height in the human brain. Proceedings of the National Academy of Sciences of the USA 2003;100:10038-42.

6 Peretz I, Coltheart M. Modularity of music processing. Nat Neurosci 2003;6:688-91.

7 Koelsch S, Siebel WA. Towards a neural basis of music perception. Trends Cogn Sci 2005;9:578-84.

8 Griffiths TD, Rees A, Green GGR. Disorders of human complex sound processing. Neurocase 1999;5:365-78.

9 Peretz I, Champod A-S, Hyde KL. Varieties of musical disorders. The Montreal Battery for Evaluation of Amusia. Ann N Y Acad Sci 2003;999:58.

10 Griffiths TD, Warren JD. The planum temporale as a computational hub. Trends Neurosci 2002;25:348-53.

11 Ayotte J, Peretz I, Rousseau I, Bard C, Bojanowski M. Patterns of music agnosia associated with middle cerebral artery infarcts. Brain 2000;123:1926-38.

12 Satoh M, Takeda K, Nagata K, Shimosegawa E, Kuzuhara S. Positronemission tomography of brain regions activated by recognition of familiar music. Am J Neuroradiol 2006;27:1101-6.

13 Rohrer JD, Knight WD, Warren JE et al. Word-finding difficulty: a clinical analysis of the progressive aphasias. Brain 2007; doi:10.1093/brain/awm251.

14 Janata P, Birk JL, Van Horn JD et al. The cortical topography of tonal structures underlying Western music. Science 2002;298:2167-70.

15 Maess B, Koelsch S, Gunter TC, Friederici AD. Musical syntax is processed in Broca's area: an MEG study. Nat Neurosci 2001;4:540-5.

16 Liegeois-Chauvel C, Peretz I, Babai M, Laguittin V, Chauvel P. Contribution of different cortical areas in the temporal lobes to music processing. Brain 1998;121:1853-67.

17 Schuppert M, Munte TF, Wieringa BM, Altenmuller E. Receptive amusia: evidence for cross-hemispheric neural networks underlying music processing strategies. Brain 2000;123:546-59.

18 Samson S, Ehrle N, Baulac M. Cerebral substrates for musical temporal processes. Ann N Y Acad Sci 2001;930:166-78.

19 Sakai K, Hikosaka O, Miyauchi S et al. Neural representation of a rhythm depends on its interval ratio. J Neurosci 1999;19:10074-81.

20 Samson S, Zatorre RJ, Ramsay JO. Deficits of musical timbre perception after unilateral temporal-lobe lesion revealed with multidimensional scaling. Brain 2002;125:511-23.

21 Kohlmetz C, Muller SV, Nager W, Munte TF, Altenmuller E. Selective loss of timbre perception for keyboard and percussion instruments following a right temporal lesion. Neurocase 2003;9:86-93.

22 Blood AJ, Zatorre RJ. Intensely pleasurable responses to music correlate with activity in brain regions implicated in reward and emotion. Proc Natl Acad Sci 2001;98:11818-23.

23 Griffiths TD, Warren JD, Dean JL, Howard D. 'When the feeling's gone': a selective loss of musical emotion. J Neurol Neurosurg Psychiatry 2004;75:344-5.

24 Gosselin N, Peretz I, Noulhiane M et al. Impaired recognition of scary music following unilateral temporal lobe excision. Brain 2005;128:628-40. 